\title{
Modifying the spectral weights of vibronic transitions via strong coupling to surface plasmons - Supporting Information
}

Rahul Deshmukh ${ }^{1,2}$, Paulo Marques, Anurag Panda ${ }^{3}$, Matthew Y. Sfeir ${ }^{2,4}$, Stephen R. Forrest ${ }^{3,5^{*}}$, Vinod M. Menon ${ }^{1,2 *}$

${ }^{1}$ Department of Physics, City College of the City University of New York (CUNY), New York, NY 10031, USA

${ }^{2}$ Department of Physics, Graduate Center of the City University of New York (CUNY), New York, NY 10016, USA

${ }^{3}$ Department of Materials Science and Engineering, University of Michigan, Ann Arbor, Michigan 48109, USA

${ }^{4}$ Photonics Initiative, Advanced Science Research Center, City University of New York, New York, NY 10031, USA

${ }^{5}$ Department of Electrical Engineering and Computer Science and Physics, University of Michigan, Ann Arbor, Michigan 48109, USA

* Email: stevefor@umich.edu ; vmenon@ccny.cuny.edu

S1: Sample Fabrication

S2: Hopfield Coefficients

S3: Weak coupling with 3nm DIP

Figures:

Fig. S1: Ellipsometry data and AFM image for horizontally oriented (flat-lying) DIP molecules.

Fig. S2: Hopfield coefficients for the polariton branches of DIP strongly coupled with SPP.

Fig. S3a and Fig. S3b: Reflectivity data for 3nm of DIP on Ag showing weak coupling. 


\section{S1. Sample Fabrication}

The silver samples were fabricated on No. 2 glass coverslips from Fisher Scientific. The coverslips were cleaned using acetone, methanol and iso propyl alcohol wash and dried with nitrogen gas. The clean glass coverslips were then introduced into the vacuum chamber of an electron beam evaporator (AJA International) with the vacuum at less than $10^{-7}$ Torr. First, a $2 \mathrm{~nm}$ Ge wetting layer was deposited at $0.2 \AA / \mathrm{s}$ followed by $\mathrm{Ag}$ deposition at $0.8 \AA-1 \AA / \mathrm{s}$. Finally, a $3 \mathrm{~nm}$ Al layer was deposited at $0.5 \AA / \mathrm{s}$ which acted as a spacer to isolate the Ag from the DIP deposited later and also protected the $\mathrm{Ag}$ from oxidation.

The diindenoperylene (DIP) was obtained commercially synthesized from Lumtech and purified once using the vacuum thermal gradient method. The DIP layer was grown by thermal evaporation at $3 \AA / \mathrm{s}$ at a vacuum of $2 \times 10^{-7}$ Torr. The molecules tend to orient themselves vertically on dielectric surfaces under these conditions. To obtain horizontally oriented DIP molecules, a wetting layer of PTCDA is deposited first. The ellipsometry data for horizontally oriented DIP molecules is shown in Fig. S1. In this case, the large anisotropy arises from the strong absorption peaks and corresponding changes in the real component of the ordinary refractive index.

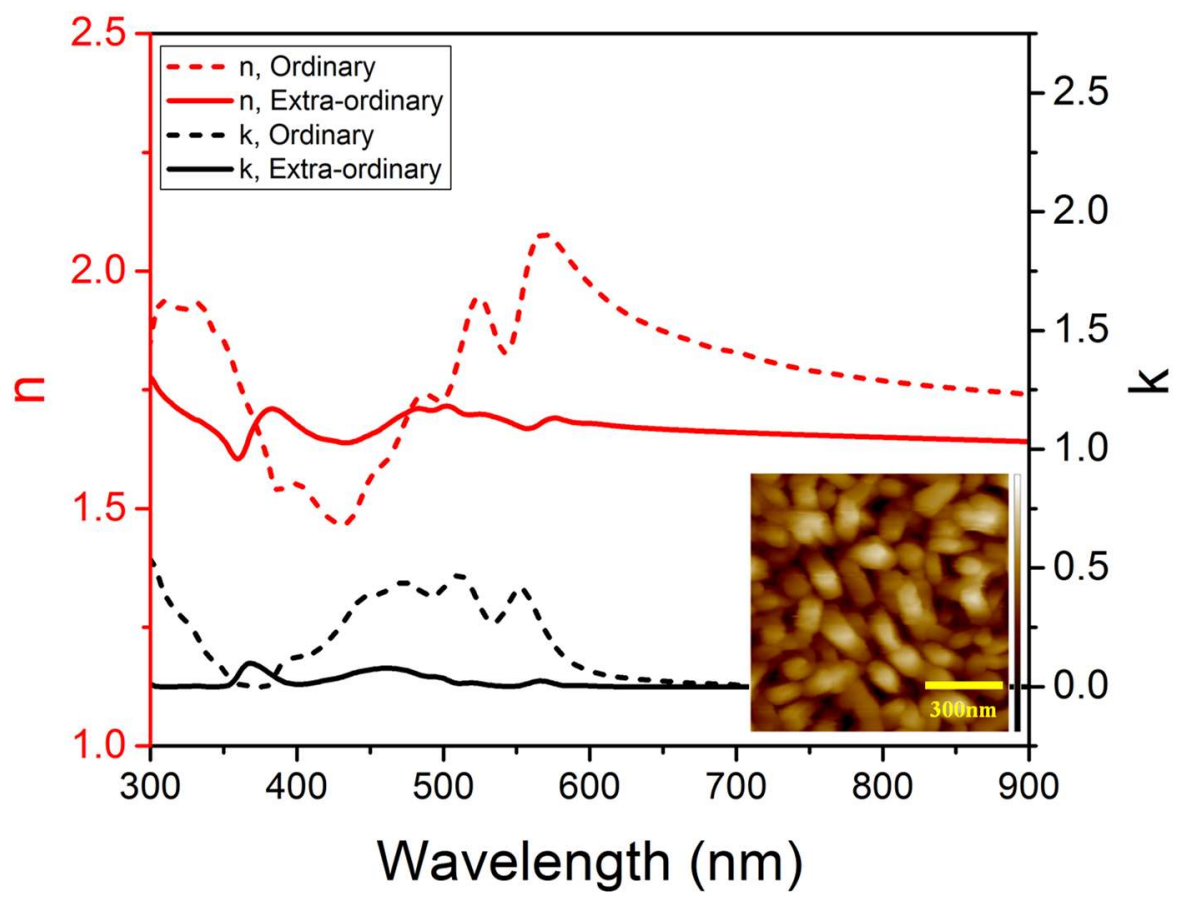

Figure S1. Ellipsomeric data showing the ordinary and extraordinary refractive index (real and imaginary parts) for a flat lying DIP film. (Inset) AFM image showing the surface profile of the flat film. 


\section{S2. Hopfield Coefficients}

We model the full coupled system of one surface plasmon polariton mode and 3 vibronic transitions using a $4 \times 4$ Hamiltonian. The coupled equation is written as

$$
\left(\begin{array}{cccc}
E_{S P P}(\theta) & V_{1} & V_{2} & V_{3} \\
V_{1} & E_{1} & 0 & 0 \\
V_{2} & 0 & E_{2} & 0 \\
V_{3} & 0 & 0 & E_{3}
\end{array}\right)\left(\begin{array}{l}
\alpha \\
\beta \\
\gamma \\
\delta
\end{array}\right)=\varepsilon\left(\begin{array}{l}
\alpha \\
\beta \\
\gamma \\
\delta
\end{array}\right)
$$

Here $\mathrm{E}_{S P P}$ is the energy for the surface plasmon polariton (SPP) at a given angle and $\mathrm{E}_{1}, \mathrm{E}_{2}$, and $\mathrm{E}_{3}$ refer to the three vibronic transitions of DIP. $V_{1}, V_{2}$, and $V_{3}$ are the interaction potentials which manifest as Rabi splits in the experiment. $\alpha, \beta, \gamma$ and $\delta$ are the coefficients for the new eigen vectors (polariton states) for the hybrid system. They directly give the fractional contribution of the individual transitions and the SPP in each new polariton branch. Once $E_{1}, E_{2}$, and $E_{3}$ are assigned fixed values from ellipsometry data of DIP, this coupled system is solved and fit to the experimental polaritonic branches to extract the values of the Hopfield Coefficients for each angle (shown in Fig. S2) and the individual Rabi splits.

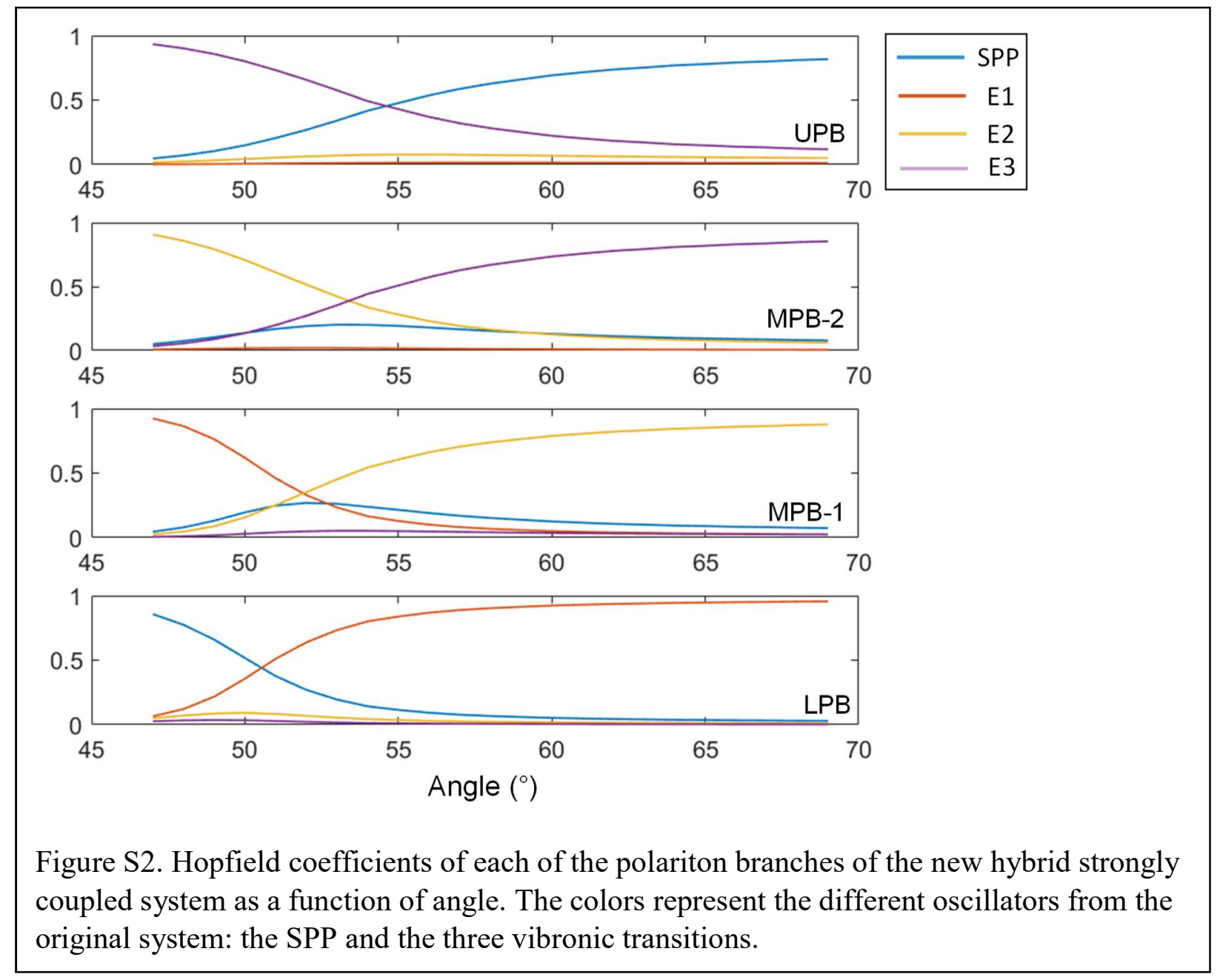




\section{S3. Weak coupling with $3 \mathrm{~nm}$ DIP}

To establish that the strong coupling of DIP with the Ag is responsible for the change in PL spectra, we prepared additional control samples of $3 \mathrm{~nm}$ DIP on Ag-on-glass as well as $\mathrm{Si}$ substrates. Angle resolved reflectivity was performed on the Ag sample using Kretschmann configuration and the results are shown in Fig. S3. There is no avoided crossing that can be observed. Rather the dips in reflection follow that of a bare Ag surface plasmon, indicating weak coupling with the excitons of DIP.
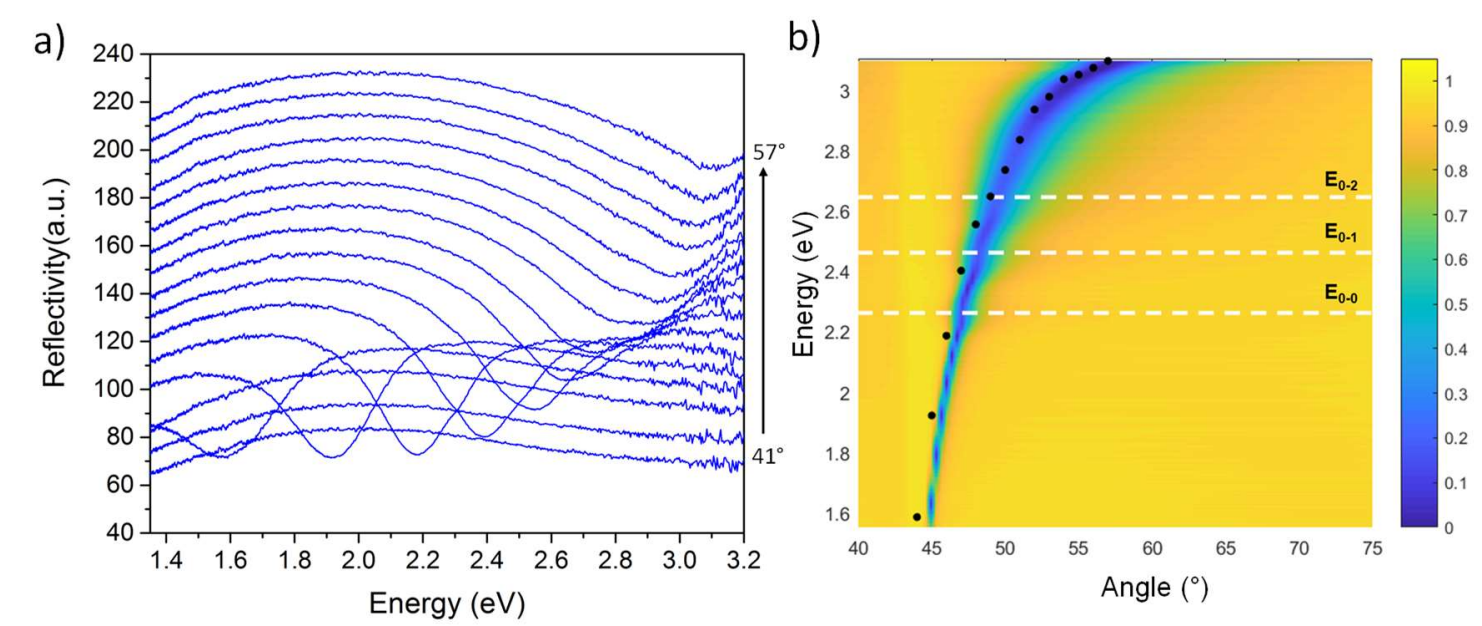

Figure S3: a) Angle resolved reflectivity for $3 \mathrm{~nm}$ of DIP on Ag. The absence of avoided crossings show that the DIP is only weakly coupled to the Ag. b) Simulated results (color plot) and experimental data from a) (black dots). 